\title{
The Detour Polynomials of the Corona of Graphs
}

\author{
Ali Aziz Ali \\ aliazizali1933@yahoo.com \\ Academic Professor \\ University of Mosul, Mosul, Iraq
}

\section{Gashaw A. Muhammed-Saleh}

gashaw.mohammed@su.edu.krd

College of Science

University of Salahaddin, Erbil, Iraq

Received on: 05/04/2012

Accepted on: 28/06/2012

\begin{abstract}
A new graph distance based polynomial, called detour polynomial, is introduced. The detour polynomial and the detour index of the corona $G_{1} \circ G_{2}$ of two connected disjoint graphs $G_{1}$ and $G_{2}$ are obtained in this paper.

Keywords: distance, detour distance, detour polynomial, detour index, corona.

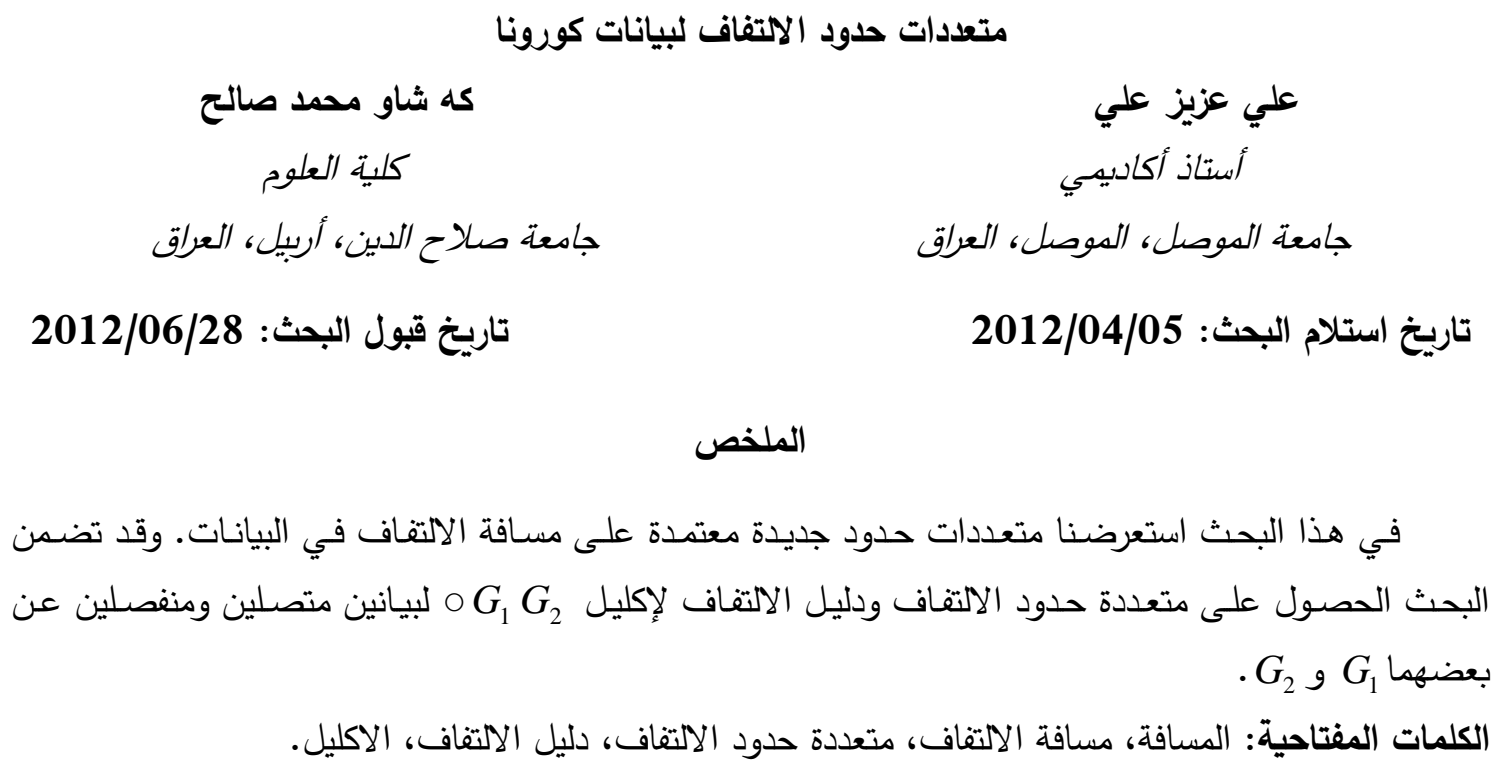

\section{Introduction :}

The concept of Hosoya polynomial was first put forward in 1988 by Hosoya [14]. Several authors, such as [1]-[4], [12], and [14]-[17] had obtained Hosoya polynomials for special graphs, graphs having some kind of regularity and for compound graphs obtained by using some well-known binary operations in graph theory.

In this paper, we consider finite connected graphs without loops or multiple edges. For undefined concepts and notations see [6] and [13].

The standard distance $d(u, v)$ between two vertices $u$ and $v$ in a connected graph $G$ is the length of a shortest $u-v$ path in $G$. This is not the only way, however, that distance has been defined on the vertex set of a connected graph. The length of a longest $u-v$ path between two vertices $u$ and $v$ in a connected graph is called the detour distance $D(u, v)$ between $u$ and $v$. As with standard distance, detour distance is a metric on the vertex set of any connected graph [7]. A $u-v$ path of length $D(u, v)$ is $u-v$ detour. It is clear that $D(u, v)=1$ if and only if $u v$ is a bridge of $G$, and $D(u, v)=p(G)-1$ if and only if $G$ contains a Hamiltonian $u-v$ path. Furthermore, 
$d(u, v)=D(u, v)$ for every two vertices $u$ and $v$ of $G$ if and only if $G$ is a tree. It is possible, however, that $d(u, v)=D(u, v)$ for some pairs $u, v$ of distinct vertices in a graph that contains no bridges. The detour eccentricity $e_{D}(v)$ of a vertex $v$ is the maximum detour distances from $v$ to all other vertices in $G$. The detour radius $\operatorname{rad}_{D}(G)$ of a connected graph $G$ is the minimum detour eccentricity among the vertices of $G$, and the detour diameter $\operatorname{diam}_{D}(G)$ is the maximum detour eccentricity among the vertices of $G$. Since $d(x, y) \leq D(x, y)$ for every two vertices $x$ and $y$ in $G$, it follows that $e(v) \leq e_{D}(v)$ for every vertex $v$.Therefore, $\operatorname{rad}(G) \leq \operatorname{rad}_{D}(G)$ and $\operatorname{diam}(G) \leq \operatorname{diam}_{D}(G)$ for every connected graph $G$ ( see [9], [10] and [18]). In fact $\operatorname{rad}_{D}(G) \leq \operatorname{diam}_{D}(G) \leq 2 \operatorname{rad}_{D}(G)$, see [9].

Let $G$ be a connected graph of order $p$ and size $q$. The detour polynomial of $G$, denoted by $D(G ; x)$, is defined by

$$
D(G ; x)=\sum_{u, v \in V} x^{D(u, v)}, u \neq v ; V=V(G) .
$$

It is clear that $D(G ; x)=\sum_{k \geq 1} C_{D}(G, k) x^{k}$, in which $C_{D}(G, k)$ is the number of unordered pairs u, v such that $D(u, v)=k$.

Amić and Trinajstić [5] were first to consider the detour index $d d(G)$ defined as the sum of the detour matrix elements above the main diagonal. The detour index is used in quantitative structure-activity relationship (QSAR) studies. Lukovits [15] tested the detour index on the correlation of the boiling points of alkanes of cycloalkanes. The detour index can also be obtained from the detour polynomial, because

$$
d d(G)=\sum_{u, v} D(u, v)=\left.\frac{d D(G ; x)}{d x}\right|_{x=1}=\sum_{k \geq 1} k C_{D}(G, k) .
$$

Let $C_{D}(G ; v, k)$ be the number of vertices $u(\neq v)$ such that $D(u, v)=k$. Then, we define for each vertex $v$ of $G$ :

$$
D(G ; v, x)=\sum_{k \geq m(v)}^{e_{D}(v)} C_{D}(G ; v, k) x^{k}=\sum_{\substack{u \in V \\ u \neq v}} x^{D(v, u)},
$$

where $e_{D}(v)$ is the detour eccentricity of vertex $v$, and $m(v)$ is the minimum detour distance from $v$. This polynomial is called the detour polynomial of vertex $v$. It is clear that

$$
D(G ; x)=\frac{1}{2} \sum_{v \in V} D(G ; v, x) .
$$

\section{The Corona $G_{1} \circ G_{2}$ :}

The corona of two disjoint graphs $G_{1}$ and $G_{2}$ of orders $p_{1}$ and $p_{2}$, respectively, is the graph $G=G_{1} \circ G_{2}$ defined by taking one copy of $G_{1}$ and $p_{1}$ copies of $G_{2}$, and then joining the $i^{\text {th }}$ vertex of $G_{1}$ to every vertex in the $i^{\text {th }}$ copy of $G_{2}$, as illustrated in Fig.2.1, where the copies of $G_{2}$ are denoted by $G_{1}^{\prime}, G_{2}^{\prime}, \ldots, G_{p_{1}}^{\prime}$,

$$
\begin{aligned}
& V_{1}=V\left(G_{1}\right)=\left\{v_{1}, v_{2}, \ldots, v_{p_{1}}\right\}, \\
& U^{(i)}=V\left(G_{i}^{\prime}\right)=\left\{u_{1}^{(i)}, u_{2}^{(i)}, \ldots, u_{p_{2}}^{(i)}\right\}, \text { for } i=1,2, \ldots, p_{1},
\end{aligned}
$$


$V_{2}=V\left(G_{2}\right)=\left\{u_{1}, u_{2}, \ldots, u_{p_{2}}\right\}$,

and

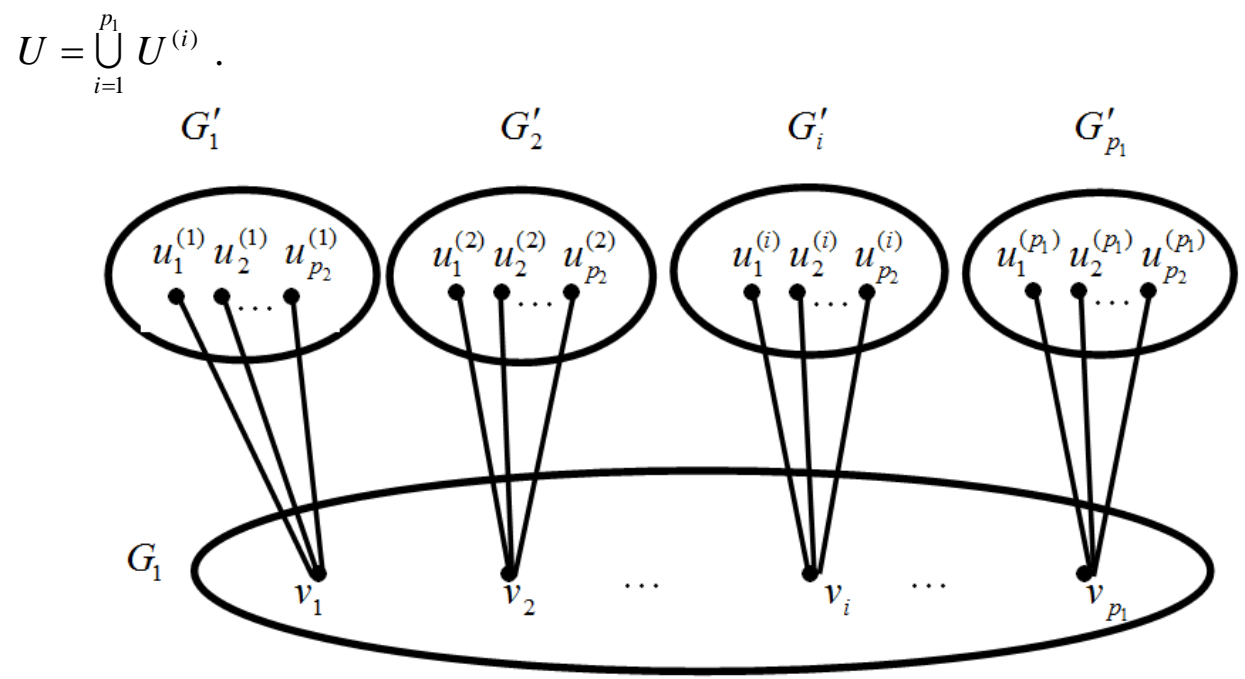

Fig.2.1 The corona $G_{1} \circ G_{2}$

It follows, from the definition of the corona $G_{1} \circ G_{2}$, that $p\left(G_{1} \circ G_{2}\right)=p_{1}\left(1+p_{2}\right), q\left(G_{1} \circ G_{2}\right)=q_{1}+p_{1} q_{2}+p_{1} p_{2}$ and $\operatorname{diam}\left(G_{1} \circ G_{2}\right)=\operatorname{diam}\left(G_{1}\right)+2$.

Note that $G_{1} \circ G_{2} \neq G_{2} \circ G_{1}$ unless $G_{1} \cong G_{2}$.

Thus, the corona is a binary graph operation, it was defined for the first time by Frucht and Harary [11] in 1970, and used in studying the automorphism group of graphs. Recently, in 2007, the Hosoya polynomial of the corona of two graphs, with respect to Steiner distance, was obtained [1]. So, we think that it is an interesting to obtain the Hosoya polynomial of the corona with respect to detour distance.

We begin discussing the detour distance and detour diameter of $G_{1} \circ G_{2}$. Let $e_{D}^{(i)}(w)$ be the detour eccentricity of vertex w in the graph $G_{i}, \mathrm{i}=1,2$. Define the graph $G_{2}^{+}$as $G_{2}+K_{1}$.

Proposition 2.1: Let $w_{1}$ and $w_{2}$ be any two distinct vertices of $G=G_{1} \circ G_{2}$. Then, the detour distance $D_{G}\left(w_{1}, w_{2}\right)$ equals:

1) $D_{G_{1}}\left(w_{1}, w_{2}\right)$, if $w_{1}, w_{2} \in V\left(G_{1}\right)$;

2) $D_{G_{2}^{+}}\left(w_{1}, w_{2}\right)$, if $w_{1}, w_{2} \in V\left(G_{i}^{\prime}\right)$ for $1 \leq i \leq p_{1}$;

3) $1+e_{D}^{(2)}\left(w_{2}\right)$, if $w_{1}=v_{i}$ and $w_{2} \in V\left(G_{i}^{\prime}\right)$ for some $i, 1 \leq i \leq p_{1}$;

4) $D_{G_{1}}\left(v_{i}, v_{j}\right)+e_{D}^{(2)}\left(w_{2}\right)+1$, if $w_{1}=v_{i}$ and $w_{2} \in V\left(G_{j}^{\prime}\right)$, where $i \neq j$ and $1 \leq i, j \leq p_{1}$;

5) $D_{G_{1}}\left(v_{i}, v_{j}\right)+e_{D}^{(2)}\left(w_{1}\right)+e_{D}^{(2)}\left(w_{2}\right)+2$, if $\quad w_{1} \in V\left(G_{i}^{\prime}\right), w_{2} \in V\left(G_{j}^{\prime}\right)$, where $i \neq j$ and $1 \leq i, j \leq p_{1}$.

Proof: 1) Obvious .

2) Any detour between two vertices of $G_{i}^{\prime}, 1 \leq i \leq p_{1}$ must contain vertex $v_{i}$ and does not contain another vertex of $G_{1}$. Therefore $D_{G}\left(w_{1}, w_{2}\right)=D_{G_{2}^{+}}\left(w_{1}, w_{2}\right)$. 
3) If $w_{2}^{\prime}$ is the detour eccentric vertex of $w_{2}$ in $G_{i}^{\prime}$, that is $D_{G_{2}}\left(w_{2}, w_{2}^{\prime}\right)=e_{D}^{(2)}\left(w_{2}\right)$, then a detour from $v_{i}$ to $w_{2}$ consists of the edge $v_{i} w_{2}^{\prime}$ followed by the detour in $G_{i}^{\prime}$ between $w_{2}^{\prime}$ to $w_{2}$. Therefore $D_{G}\left(w_{1}, w_{2}\right)=1+e_{D}^{(2)}\left(w_{2}\right)$.

4) In this case a detour between $w_{1}$ and $w_{2}$ in $G$ consists of a detour between $v_{i}$ and $v_{j}$ in $G_{1}$ followed by the edge $v_{i} w_{2}^{\prime}$ then the detour between $w_{2}^{\prime}$ and $w_{2}$ in $G_{j}^{\prime}$, here $w_{2}^{\prime}$ is the detour eccentric vertex of $w_{2}$ in $G_{j}^{\prime}$. Thus

$$
D_{G}\left(w_{1}, w_{2}\right)=D_{G_{1}}\left(v_{i}, v_{j}\right)+1+e_{D}^{(2)}\left(w_{2}\right) .
$$

5) A detour between a vertex $w_{1}$ of $G_{i}^{\prime}$ and a vertex $w_{2}$ of $G_{j}^{\prime}, i \neq j$, is constructed from a detour from $w_{1}$ to $w_{1}^{\prime}$ ( the detour eccentric vertex of $w_{1}$ ), followed by the edge $w_{2}^{\prime} v_{j}$, then a detour from $v_{i}$ to $v_{j}$ in $G_{1}$, then followed by the edge $v_{j} w_{2}^{\prime}$ and finally followed by a detour $w_{2}^{\prime}-w_{2}$ in $G_{j}^{\prime}$. The length of this detour is $D_{G_{1}}\left(v_{i}, v_{j}\right)+e_{D}^{(2)}\left(w_{1}\right)+e_{D}^{(2)}\left(w_{2}\right)+2$. Hence, the proof is complete.

Proposition 2.2: For a connected nontrivial graph $G_{1}$ and any connected graph $G_{2}$, $\operatorname{diam}_{D}\left(G_{1} \circ G_{2}\right)=\operatorname{diam}_{D}\left(G_{1}\right)+2 \operatorname{diam}_{D}\left(G_{2}\right)+2$.

Proof: Let $v_{i}, v_{j}$ be two vertices of $G_{1}$ such that $D_{G_{1}}\left(v_{i}, v_{j}\right)=\operatorname{diam}_{D}\left(G_{1}\right)$; and let $u_{l}$, $u_{k}$ be two vertices of $G_{2}$ such that $D_{G_{2}}\left(u_{l}, u_{k}\right)=\operatorname{diam}_{D}\left(G_{2}\right)$.

One may easily check from Fig.2.1 and Proposition 2.1(5), that

$$
\begin{gathered}
D_{G}\left(u_{l}^{(i)}, u_{k}^{(j)}\right)=D_{G_{i}^{\prime}}\left(u_{l}^{(i)}, u_{k}^{(i)}\right)+1+D_{G_{1}}\left(v_{i}, v_{j}\right)+1+D_{G_{j}^{\prime}}\left(u_{l}^{(j)}, u_{k}^{(j)}\right) \\
=\operatorname{diam}_{\mathrm{D}}\left(G_{1}\right)+2 \operatorname{diam}_{\mathrm{D}}\left(G_{2}\right)+2 .
\end{gathered}
$$

Now, let $w_{1}, w_{2}$ be any two vertices of $G_{1} \circ G_{2}$, then:

1- If $w_{1}, w_{2} \in V\left(G_{1}\right)$, then $D_{G}\left(w_{1}, w_{2}\right) \leq \operatorname{diam}_{D}\left(G_{1}\right)$;

2- If $w_{1}, w_{2} \in V\left(G_{r}^{\prime}\right)$ for some $r, 1 \leq r \leq p_{1}$, then, let $\mathrm{Q}$ be a $w_{1}-w_{2}$ detour. If $v_{r} \notin V(Q)$, then $D_{G}\left(w_{1}, w_{2}\right) \leq \operatorname{diam}_{D}\left(G_{2}\right)$.

If $v_{r} \in V(Q)$, then $Q-v_{r}$ consists of two paths in $G_{r}^{\prime}$ each of length not more than $\operatorname{diam}_{D}\left(G_{2}\right)$. Thus $D_{G}\left(w_{1}, w_{2}\right) \leq 2 \operatorname{diam}_{D}\left(G_{2}\right)+2$;

3- If $w_{1} \in V\left(G_{t}^{\prime}\right)$ and $w_{2} \in V\left(G_{s}^{\prime}\right), t \neq s$, then $D_{G}\left(w_{1}, w_{2}\right) \leq \operatorname{diam}_{D}\left(G_{1}\right)+2 \operatorname{diam}_{D}\left(G_{2}\right)+2 ;$

4- If $w_{1} \in V\left(G_{1}\right)$ and $w_{2} \in V\left(G_{m}^{\prime}\right)$, then $D_{G}\left(w_{1}, w_{2}\right) \leq \operatorname{diam}_{D}\left(G_{1}\right)+1+\operatorname{diam}_{D}\left(G_{2}\right)$.

Therefore, for all cases of $w_{1}$ and $w_{2}$, we have

$$
D_{G}\left(w_{1}, w_{2}\right) \leq \operatorname{diam}_{D}\left(G_{1}\right)+2 \operatorname{diam}_{D}\left(G_{2}\right)+2 .
$$

Hence, the proof is complete.

The minimum detour of a connected graph $\mathrm{G}$ is denoted by $D_{\min }(G)$, and defined as

$$
D_{\min }(G)=\min \{D(u, v): u \neq v, u, v \in V(G)\} .
$$


Let $H_{1}$ and $H_{2}$ be disjoint connected graphs, and let $u_{1}$ and $u_{2}$ be vertices of $H_{1}$ and $H_{2}$, respectively. Then, the vertex identified graph $H_{1} \bullet H_{2}$ is obtained from $H_{1}$ and $H_{2}$ by identifying the vertices $u_{1}$ and $u_{2}$. We notice, that

$D_{\text {min }}\left(H_{1} \bullet H_{2}\right)=\min \left\{D_{\min }\left(H_{1}\right), D_{\text {min }}\left(H_{2}\right)\right\}$.

Applying this fact, we can easily prove the following proposition, which determines $D_{\text {min }}\left(G_{1} \circ G_{2}\right)$.

Proposition 2.3 : For disjoint connected nontrivial graphs $G_{1}$ and $G_{2}$, we have

$$
D_{\text {min }}\left(G_{1} \circ G_{2}\right)=\min \left\{D_{\min }\left(G_{1}\right), D_{\min }\left(G_{2}^{+}\right)\right\} .
$$

\section{The Detour Polynomial of $G_{1} \circ G_{2}$ :}

To determine the detour polynomial of the corona $G_{1} \circ G_{2}$, we introduce the detour eccentric polynomial of a connected graph $G$, defined as follows:

For example:

$$
D_{e c c}(G ; x)=\sum_{v \in V(G)} x^{e_{D}(v)} .
$$

$$
\begin{aligned}
& D_{e c c}\left(C_{p} ; x\right)=p x^{p-1} \\
& D_{e c c}\left(P_{p} ; x\right)= \begin{cases}2 \sum_{i=1}^{\frac{p}{2}} x^{p-i}, & \text { for even } p \\
x^{\frac{p-1}{2}}+2 \sum_{i=1}^{\frac{p-1}{2}} x^{p-i} & \text { for odd } p .\end{cases}
\end{aligned}
$$

We shall obtain the detour polynomial of the corona $G_{1} \circ G_{2}$ in terms of $D\left(G_{i} ; x\right)$ and $D_{\text {ecc }}\left(G_{i} ; x\right), i=1,2$.

We set the following definitions:

$$
\begin{aligned}
D\left(G, V_{1} ; x\right) & =\sum_{i=1}^{p_{1}} D\left(G, v_{i} ; x\right), \\
D\left(G, U^{(i)} ; x\right) & =\sum_{j=1}^{p_{2}} D\left(G, u_{j}^{(i)} ; x\right), \\
D(G, U ; x) & =\sum_{i=1}^{p_{1}} D\left(G, U^{(i)} ; x\right) .
\end{aligned}
$$

Then

$$
D(G ; x)=\frac{1}{2}\left[D\left(G, V_{1} ; x\right)+D(G, U ; x)\right] .
$$

\section{Proposition 3.1:}

$$
D\left(G, V_{1} ; x\right)=2 D\left(G_{1} ; x\right)+x p_{1} D_{e c c}\left(G_{2} ; x\right)+2 x D_{e c c}\left(G_{2} ; x\right) D\left(G_{1} ; x\right) .
$$

Proof: From Proposition 2.1, for each $v_{i} \in V\left(G_{1}\right)$, we have

$$
D\left(G, v_{i} ; x\right)=\sum_{\substack{j=1 \\ j \neq i}}^{p_{1}} x^{D\left(v_{i}, v_{j}\right)}+\sum_{w \in U^{(i)}} x^{D\left(v_{i}, w\right)}+\sum_{\substack{w \in U^{(j)} \\ j \neq i}}^{p_{1}} x^{D\left(v_{i}, w\right)} .
$$

Summing over all $i=1,2, \ldots, p_{1}$, we get 


$$
\begin{aligned}
D\left(G, V_{1} ; x\right)= & \sum_{\substack{i, j \in V_{1} \\
i \neq j}} x^{D_{G_{1}}\left(v_{i}, v_{j}\right)}+p_{1} \sum_{w \in V_{2}} x^{1+e_{G 2}(w)}+\sum_{\substack{i=1 \\
p_{1}}} \sum_{\substack{w \in U^{(j)} \\
i \neq j}} x^{1+D_{G_{1}}\left(v_{i}, v_{j}\right)+e_{G 2}(u)} \\
& =2 D\left(G_{1} ; x\right)+p_{1} x D_{e c c}\left(G_{2} ; x\right)+x D_{e c c}\left(G_{2} ; x\right) \cdot 2 D\left(G_{1} ; x\right) .
\end{aligned}
$$

\section{Proposition 3.2:}

$$
\begin{aligned}
D(G, U ; x)= & 2 p_{1} D\left(G_{2}^{+} ; x\right)-p_{1} x D_{e c c}\left(G_{2} ; x\right)+x D_{e c c}\left(G_{2} ; x\right) .2 D\left(G_{1} ; x\right) \\
& +x^{2}\left[D_{e c c}\left(G_{2} ; x\right)\right]^{2} .2 D\left(G_{1} ; x\right) .
\end{aligned}
$$

Proof: From Proposition 2.1, for each $u_{i}^{(j)}, i=1,2, \ldots, p_{2}$ and $j=1,2, \ldots, p_{1}$, we have

$$
D\left(G, u_{i}^{(j)} ; x\right)=D\left(G_{2}^{+} ; u_{i}^{(j)} ; x\right)+\sum_{\substack{k=1 \\ k \neq j}}^{p_{1}} x^{D_{G_{1}}\left(v_{j}, v_{k}\right)+1+e e c_{D}\left(u_{i}\right)}+\sum_{\substack{n=1, k=1 \\ n \neq j}}^{n=p_{1}, p_{2}=k} x^{2+e c c_{D}\left(u_{i}\right)+e c c_{D}\left(u_{k}\right)+D_{G_{1}}\left(v_{j}, v_{n}\right)} .
$$

Summing over both $j=1,2, \ldots, p_{1}$ and $i=1,2, \ldots, p_{2}$, we get, using the above notations and definitions:

$$
\begin{aligned}
D(G, U ; x) & =p_{1}\left[2 D\left(G_{2}^{+} ; x\right)-x D_{e c c}\left(G_{2} ; x\right)\right]+x D_{e c c}\left(G_{2} ; x\right) \cdot 2 D\left(G_{1} ; x\right) \\
& +x^{2} D_{e c c}\left(G_{2} ; x\right) \cdot D_{e c c}\left(G_{2} ; x\right) \cdot 2 D\left(G_{1} ; x\right) .
\end{aligned}
$$

Hence, the proof is complete.

Theorem 3.3: For connected nontrivial graphs $G_{1}$ and $G_{2}$, the detour polynomial of the corona graph $G_{1} \circ G_{2}$ is

$$
D\left(G_{1} \circ G_{2} ; x\right)=\left[1+x D_{e c c}\left(G_{2} ; x\right)\right]^{2} \cdot D\left(G_{1} ; x\right)+p_{1} D\left(G_{2}^{+} ; x\right) .
$$

Proof: From Propositions 3.1 and 3.2, we obtain

$$
\begin{aligned}
D\left(G_{1} \circ G_{2} ; x\right)= & \frac{1}{2}\left[2 D\left(G_{1} ; x\right)+p_{1} x D_{e c c}\left(G_{2} ; x\right)+2 x D_{e c c}\left(G_{2} ; x\right) D\left(G_{1} ; x\right)\right. \\
& +2 p_{1} D\left(G_{2}^{+} ; x\right)-p_{1} x D_{e c c}\left(G_{2} ; x\right)+2 x D_{e c c}\left(G_{2} ; x\right) \cdot D\left(G_{1} ; x\right) \\
+ & \left.2 x^{2} D\left(G_{1} ; x\right)\left[D_{e c c}\left(G_{2} ; x\right)\right]^{2}\right] \\
= & D\left(G_{1} ; x\right)+2 x D_{e c c}\left(G_{2} ; x\right) \cdot D\left(G_{1} ; x\right)+p_{1} D\left(G_{2}^{+} ; x\right) \\
+ & x^{2}\left[D_{e c c}\left(G_{2} ; x\right)\right]^{2} \cdot D\left(G_{1} ; x\right) \\
= & {\left[1+x D_{e c c}\left(G_{2} ; x\right)\right]^{2} \cdot D\left(G_{1} ; x\right)+p_{1} D\left(G_{2}^{+} ; x\right) . }
\end{aligned}
$$

To obtain the detour index of the corona $G_{1} \circ G_{2}$, we give the definition of the eccentricity sum of a graph, denoted by $S_{e c c}(G)$ :

$$
S_{e c c}(G)=\sum_{v \in V(G)} e c c_{D}(v) .
$$

Corollary 3.4: The detour index of $G_{1} \circ G_{2}$ is given as

$$
d d\left(G_{1} \circ G_{2}\right)=\left(1+p_{2}\right)^{2} \cdot d d\left(G_{1}\right)+p_{1}\left(p_{1}-1\right)\left(p_{2}+1\right)\left[p_{2}+S_{e c c}\left(G_{2}\right)\right]+p_{1} \cdot d d\left(G_{2}^{+}\right),
$$

Proof: Taking the derivative of $D\left(G_{1} \circ G_{2} ; x\right)$ given in Theorem 3.3, with respect to $x$, we get

$$
\begin{aligned}
D^{\prime}\left(G_{1} \circ G_{2} ; x\right) & =\left[1+x D_{e c c}\left(G_{2} ; x\right)\right]^{2} \cdot D^{\prime}\left(G_{1} ; x\right) . \\
+ & 2\left[1+x D_{e c c}\left(G_{2} ; x\right)\right] \cdot\left[D_{e c c}\left(G_{2} ; x\right)+x D_{e c c}^{\prime}\left(G_{2} ; x\right)\right] \cdot D\left(G_{1} ; x\right) \\
+ & p_{1} D^{\prime}\left(G_{2}^{+} ; x\right)
\end{aligned}
$$


Then, putting $\mathrm{x}=1$, we get

$$
\begin{aligned}
d d\left(G_{1} \circ G_{2}\right) & =D^{\prime}\left(G_{1} \circ G_{2} ; 1\right) \\
= & \left(1+p_{2}\right)^{2} d d\left(G_{1}\right)+2\left(1+p_{2}\right)\left[p_{2}+D_{e c c}^{\prime}\left(G_{2} ; 1\right)\right] \cdot \frac{1}{2} p_{1}\left(p_{1}-1\right)+p_{1} d d\left(G_{2}^{+}\right)
\end{aligned}
$$

Then, simplifying the above expression and noticing that

$$
D_{e c c}^{\prime}\left(G_{2} ; 1\right)=S_{e c c}\left(G_{2}\right) \text {, }
$$

we get the required formula of $d d\left(G_{1} \circ G_{2}\right)$.

Example: Let $G_{1}=C_{n}$ the cycle of order $n$, and $G_{2}=K_{m}$, the complete graph of order $m$, then

$$
\begin{aligned}
& D\left(C_{n} ; x\right)= \begin{cases}n\left(x^{n-1}+x^{n-2}+\ldots+x^{\frac{n+1}{2}}\right), & \text { for odd } n, \\
n\left(x^{n-1}+x^{n-2}+\ldots+x^{1+\frac{n}{2}}+\frac{1}{2} x^{\frac{n}{2}}\right), & \text { for even } n,\end{cases} \\
& D_{e c c}\left(K_{m} ; x\right)=m x^{m-1},
\end{aligned}
$$

and

$$
D\left(G_{2}^{+} ; x\right)=D\left(K_{m+1} ; x\right)=\frac{1}{2} m(m+1) x^{m} .
$$

Thus, using Theorem 3.3, we get

$$
\begin{aligned}
D\left(C_{n} \circ K_{m} ; x\right) & =\frac{1}{2} n m(m+1) x^{m} \\
+ & \left(1+m x^{m}\right)^{2} \cdot\left\{\begin{array}{l}
n\left(x^{n-1}+x^{n-2}+\ldots+x^{\frac{n+1}{2}}\right) \quad, \quad \text { for odd } n \\
n\left(x^{n-1}+x^{n-2}+\ldots++x^{1+\frac{n}{2}}+\frac{1}{2} x^{\frac{n}{2}}\right) \quad, \quad \text { for even } n .
\end{array}\right.
\end{aligned}
$$




\section{REFERENCES}

[1] Abdullah, H. O., Hosoya polynomials of Steiner distance of Some graphs, Ph.D. Thesis, University of Salahaddin, Erbil, Iraq, 2007.

[2] Ahmed, H. G., On Wiener Polynomials of n-Distance in Graphs, M.Sc. Thesis, Dohuk University, Dohuk, Iraq, 2007.

[3] Ali, A. A. and Ali, A. M.,Wiener polynomials of the generalized distance for some special graphs, Rafiden. J. Comp. Sc. and Maths., 3(2) (2006) 103-120.

[4] Ali, A. A. and Saeed, W. A., Wiener polynomials of the tensor product, Rafiden. J. Sc., 17(1) (2006) 67-77.

[5] Amić, D. and Trinajstić, N., On the Detour Matrix, Croat. Chem. Acta, 68 (1995) 53-62.

[6] Buckly, F. and Harary, F., Distance in Graphs, Addison-Wesley, Redwood, California. USA, 1990.

[7] Chartrand, G.; Escuadro, H. and Zhang, P., Detour distance in graphs, J. Combin. Math. Combin. Comput., 53 (2005) 75-94.

[8] Chartrand, G.; Johns, G. L. and Tian S., Detour distance in graphs, Annals of Discrete Mathematics, 55 (1993) 127-136.

[9] Chartrand, G. and Zhang, P., Distance in graphs-taking the long view, AKCE J. Graphs Combin., 1(1) (2004) 1-13.

[10] Diudea, M.; Katona, G. and Lukovits, I., Detour and Cluj-Detour indices, Croat. Chem. Acta. , 71(3) (1998) 459-471.

[11] Frucht , R. and Harary , F. , On the corona of two graphs , Aequationes Math. , 4 (1970)322-324 .

[12] Gutman, I., Some properties of the Wiener polynomial, Graph Theory Notes of New York, XXV (1993), 13-18.

[13] Harary, F., Graph Theory, Addison-Wesley, Reading, Mass, 1969.

[14] Hosoya, H., On some counting polynomials in Chemistry, Discrete Applied Mathematics, 19 (1988) 239-257.

[15] Lukovits, I., The detour index, Croat. Chem. Acta, 69 (1996) 873-882.

[16] Saeed, W. A.,Wiener polynomials of Graphs, Ph.D. Thesis, Mosul University, Mosul, Iraq, 1999.

[17] Sagan, B. E., Yeh, Y. N. and Zhang, P., The Wiener polynomial of a graph, International J. of Quantum Chemistry, 60 (1999) 959-969.

[18] Santhakumaran, A. P. and Athisayanathan, S., On the connected detour number of a graph, Journal of Prime Research in Mathematics, 5 (2009) 149-170. 